\title{
COMPACT LIE GROUPS ASSOCIATED WITH ENDOMORPHISMS OF $C^{*}$-ALGEBRAS
}

\author{
BY SERGIO DOPLICHER ${ }^{1}$ AND JOHN E. ROBERTS
}

The work described in this announcement is motivated by a long-standing problem in quantum field theory. Experimental evidence and theoretical considerations [1, 2] suggest that superselection structure is determined by the representation theory of a compact group, the gauge group of the first kind. Attempts to prove the existence of this group from the general principles of quantum field theory pinpoint the inadequacy of the classical Tannaka-Krein duality theorem for compact groups: it enables one to recognize the representation theory only when the intertwining operators are given concretely as linear operators between representation spaces. The discussion of superselection theory in algebraic quantum field theory leads not to representations of a compact group but to endomorphisms of a $C^{*}$-algebra, the $C^{*}$-algebra of local observables, and the intertwining operators intertwine these endomorphisms $[3]$. We announce here, in the setting of $C^{*}$-algebras, the basic results which have allowed us to resolve this problem.

We give conditions on an endomorphism $\rho$ of a $C^{*}$-algebra $A$ with unit and trivial centre in terms of intertwining operators for powers of this endomorphism which suffice to determine a compact Lie group $G$ and an action of a $G$-dual on $A$. It is convenient to begin by describing the $C^{*}$-systems $\{B, G, \alpha\}$ which arise if we take a cross product of $A$ by the action of the $G$-dual.

Thus, we consider a $C^{*}$-algebra $B$ carrying a faithful continuous action $\alpha$ of a compact group $G$ by automorphisms and a Hilbert space $H \subset B$ of dimension $d, 1<d<+\infty$, i.e. we have an orthonornal basis $\psi_{1}, \psi_{2}, \ldots, \psi_{d}$ of $H$ consisting of isometries satisfying

$$
\psi_{i}^{*} \psi_{j}=\delta_{i j} I, \quad \sum_{i=1}^{d} \psi_{i} \psi_{i}^{*}=I .
$$

Let $B^{\alpha}$ denote the subalgebra of the fixed points. We suppose:

(a) $H$ and $B^{\alpha}$ generate $B$;

(b) $\alpha_{g}(H)=H, g \in G$;

(c) Det $\alpha_{g} \mid H=1, g \in G$;

(d) $\left(B^{\alpha}\right)^{\prime} \cap B=\mathbf{C} I$.

The first three conditions imply that $G$ is isomorphic to a closed subgroup of $\mathrm{SU}(d)$ and, hence, is a Lie group. The final condition leads to a particularly simple class of cross products.

We first give some examples of this situation.

Received by the editors April 10, 1984.

1980 Mathematics Subject Classification. Primary 46L05, 46L40, 46L60; Secondary 43A40.

${ }^{1}$ Research supported by the Ministero della Pubblica Istruzione and CNR-GNAFA. 
(i) Our $C^{*}$-algebra $B$ always contains the Cuntz algebra $O_{d}[4]$ of order $d$ generated by the Hilbert space $H$. We therefore get minimal examples by taking $B=O_{d}$ and letting $G$ be a closed subgroup of $\mathrm{SU}(d)$ acting naturally on $O_{d}$. In these examples the fixed point algebras $B^{\alpha}$ will be denoted by $O_{G}$. $O_{G}$ has trivial relative commutant in $O_{d}$.

(ii) Let $A$ be a $C^{*}$-algebra with centre $\mathbf{C} I$, and let $\beta$ be an automorphism of $A$ with $\beta^{d}=\iota$ (the identity automorphism). The covariance algebra $A \times{ }_{\beta} \mathbf{Z}_{d}$ is generated by $A$ and a unitary $V$ implementing $\beta$. Let $\hat{\mathbf{Z}}_{d}$ be identified with the centre of $\mathrm{SU}(d)$ and let $\hat{\beta}$ be the dual action on $A \times{ }_{\beta} \mathbf{Z}_{d}$. If $\tilde{\alpha}$ is the action $\hat{\mathbf{Z}}_{d} \times \mathrm{SU}(d)$ on $A \times_{\beta} \mathbf{Z}_{d} \otimes \mathcal{O}_{d}$ given by

$$
\tilde{\alpha}_{h \times g}=\hat{\beta}_{h^{-1}} \otimes \alpha_{h g}, \quad h \in \hat{\mathbf{Z}}_{d}, g \in \mathrm{SU}(d),
$$

where $\alpha$ is the natural action of $\mathrm{SU}(d)$ on $O_{d}$, then define $B$ to be the $\tilde{\alpha}_{\hat{\mathbf{z}}_{d}}{ }^{-}$ fixed points in $A \times{ }_{\beta} \mathbf{Z}_{d} \otimes O_{d}$ and $\alpha$ the action of $\mathrm{SU}(d)$ obtained by restricting $\tilde{\alpha}$ to $B$. In other words, $B$ is generated by $A \otimes I$ and $V \otimes H$. (Constructions of this kind can be found in [5].) Now $B^{\alpha}=A \otimes O_{\mathrm{SU}(d)}$ and, as in (a), $\left(B^{\alpha}\right)^{\prime} \cap B=\mathbf{C} I$.

(iii) A very similar structure occurs in Quantum Field Theory if we look at the superselection structure defined by a field algebra $\mathcal{F}$, assumed, for the sake of this example, to be given a priori and acted on by a compact Lie group $G \subset \mathbf{S U}(d)$ of gauge automorphisms [2]. The only minor difference is that $\mathcal{F}$ is a $C^{*}$-inductive limit of local von Neumann algebras $\mathcal{F}(0)$ as 0 varies over bounded open sets in space-time, and each $\mathcal{F}(0)$ is generated as a von Neumann algebra by its fixed-point subalgebra and a Hilbert space $H \subset \mathcal{F}(0)$ of dimension $d$.

Notation. We work with the category of $C^{*}$-algebras with unit; all homomorphisms will preserve the unit. If $A$ is such a $C^{*}$-algebra and $\rho$ and $\sigma$ are endomorphisms of $\mathcal{A},(\rho, \sigma)$ will denote the subspace of intertwiners in $\mathcal{A}$ between $\rho$ and $\sigma$ :

$$
T \in(\rho, \sigma) \text { if } T \in \mathcal{A} \text { and } T \rho(A)=\sigma(A) T, A \in \mathcal{A} .
$$

$\iota$ denotes the identity automorphism of $\mathcal{A}$.

The group of all finite permutations of $\mathbf{N}$ will be denoted by $\mathbf{P}_{\infty}$, and the subgroup leaving $n+1, n+2, \ldots$ fixed will be denoted by $\mathbf{P}_{n}$. The endomorphism $\sigma$ of $\mathbf{P}_{\infty}$ is defined by

$$
\sigma p(1)=1, \quad(\sigma p)(n)=1+p(n-1), \quad n>1, p \in \mathbf{P}_{\infty} .
$$

The $n+1$-cycle $(1,2, \ldots, n+1)$ will be denoted $(n, 1)$.

Our assumptions on the $C^{*}$-systems $\{B, G, \alpha\}$ have some straightforward structural implications for the fixed-point algebra:

THEOREM 1. Let $B$ be a $C^{*}$-algebra containing a Hilbert space $H$ of finite dimension $d>1$ and let $\alpha$ be a faithful strongly continuous action of a compact group $G$ on $B$ satisfying (a)-(d) above. Let $A=B^{\alpha}$ and let $\rho$ denote the endomorphism of $A$ obtained by restricting $\rho_{H}$ to $A$, where

$$
\psi B=\rho_{H}(B) \psi, \quad \psi \in H, B \in B .
$$


Then we have the following implications for $\{\mathcal{A}, \rho\}$ : there exist a unitary representation $\varepsilon$ of $\mathbf{P}_{\infty}$ in $A$ such that

$$
\begin{gathered}
\varepsilon(\sigma p)=\rho(\varepsilon(p)), \quad p \in \mathbf{P}_{\infty}, \\
\varepsilon(1,1) \in\left(\rho^{2}, \rho^{2}\right), \\
\varepsilon(s, 1) X=\rho(X) \varepsilon(r, 1), \quad X \in\left(\rho^{r}, \rho^{s}\right), r, s \in \mathbf{N}_{0},
\end{gathered}
$$

and an isometry $R \in\left(\iota, \rho^{d}\right)$ such that

$$
\begin{aligned}
R^{*} \rho(R) & =(-1)^{d-1}(1 / d) I, \\
R R^{*} & =\frac{1}{d !} \sum_{p \in \mathbf{P}_{d}} \operatorname{sign}(p) \varepsilon(p) .
\end{aligned}
$$

Every automorphism of $B$ leaving $A$ pointwise fixed is of the form $\alpha_{g}$ for some $g \in G$.

In terms of an orthonormal basis $\psi_{i}, i=1,2, \ldots, d$, in $H$ we have

$$
\begin{aligned}
\varepsilon(p) & =\sum_{i_{1}, i_{2}, \ldots, i_{n}} \psi_{i_{1}} \psi_{i_{2}} \cdots \psi_{i_{n}} \psi_{i_{p(n)}}^{*} \cdots \psi_{i_{p(1)}}^{*}, \quad p \in \mathbf{P}_{n} \\
R & =\frac{1}{\sqrt{d !}} \sum_{p \in \mathbf{P}_{d}} \operatorname{sign}(p) \psi_{p(1)} \cdots \psi_{p(d)} .
\end{aligned}
$$

The support of $\varepsilon \mid \mathbf{P}_{n}$ corresponds to the set of Young tableaux with $n$ squares and at most $d$ rows.

If $\rho$ is an endomorphism of $\mathcal{A}$ we say that $\rho$ has permutation symmetry if there is a unitary representation $\varepsilon_{\rho}$ satisfying (2)-(4). If, in addition, we have $R_{\rho} \in\left(\iota, \rho^{d}\right)$ satisfying (5) and (6), we say that $\rho$ has permutation symmetry of dimension $d$ and satisfies the special conjugate property.

Our main result is the following converse to Theorem 1.

THEOREM 2. Let $A$ be a $C^{*}$-algebra with centre $\mathbf{C I}$ and $\rho$ an endomorphism with permutation symmetry of dimension $d>1$ satisfying the special conjugate property. Then there is a $C^{*}$-algebra $B$ containing a Hilbert space $H$ of dimension $d$, a faithful strongly continuous action of a closed subgroup $G$ of $\mathrm{SU}(d)$ on $B$ satisfying (a)-(d), and a monomorphism $\pi$ of $A$ into $B$ with $\pi(A)=B^{\alpha}, \rho_{H} \circ \pi=\pi \circ \rho$. If we require, in addition, that $\pi\left(R_{\rho}\right)=R$ and $\pi\left(\varepsilon_{\rho}(1,1)\right)=\varepsilon(1,1)$, then $G$ is uniquely determined up to conjugacy in $\mathrm{SU}(d)$.

Every compact Lie group appears as a subgroup of $\mathrm{SU}(d)$ for some $d$, so the special conjugate property leading to $G \subset \mathrm{SU}(d)$ is not an essential restriction as far as $G$ is concerned. It is, however, possible to modify the above results to include a wider class of endomorphisms $\rho$ with permutation symmetry of dimension $d$ giving just $G \subset \mathrm{U}(d)$.

The remaining theorems in this announcement were derived in the course of proving Theorem 2 and are included partly for their intrinsic interest and partly to indicate the method of proof.

The Cuntz algebra $O_{d}$ plays a special role: if $B=O_{d}$, as in example (i), we write $\theta(p)$ and $S$ for the elements defined by (7) and (8), $\sigma_{H}$ for the endomorphism defined by (1), and $\sigma$ for its restriction to $O_{G}$. 
TheOREM 3. The $C^{*}$-algebra $\mathrm{O}_{\mathrm{SU}(d)}$ is generated by $S$ and $\theta(p), p \in \mathbf{P}_{\infty}$. $\mathrm{O}_{G}$ is simple for any closed subgroup $G$ of $\mathrm{SU}(d)$. By contrast, $\mathrm{O}_{\mathrm{U}(d)}$, the $C^{*}$ algebra generated by $\theta(p), p \in \mathbf{P}_{\infty}$, has many ideals.

Now the tensor powers of the defining representation of $G \subset \mathrm{U}(d)$ are realized within $O_{d}$ on the tensor powers of $H$ and the corresponding spaces of intertwining operators are precisely the subspaces $\left(\sigma^{r}, \sigma^{s}\right)$ of $O_{G}$. When $G \subset \mathrm{SU}(d)$ the representation theory within $O_{G}$ is sufficiently rich to be regarded as a dual object for $G$. An action [6] of this $G$-dual on a $C^{*}$-algebra $A$ with unit can be described in terms of $O_{G}$. It consists of an endomorphism $\rho$ of $A$ and a monomorphism $\mu: O_{G} \rightarrow A$ with

$$
\mu \circ \sigma=\rho \circ \mu, \quad \mu\left(\sigma^{r}, \sigma^{s}\right) \subset\left(\rho^{r}, \rho^{s}\right), \quad r, s \in \mathbf{N}_{0} .
$$

In this context we can define a $C^{*}$-cross product $A \otimes_{\mu} O_{d}$ of $A$ by the action of the $G$-dual: consider the problem of finding a $C^{*}$-algebra $B$ and a commutative diagram

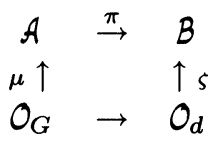

such that $\zeta(\psi) \pi(A)=\pi \circ \rho(A) \zeta(\psi), \psi \in H$, where the morphism $O_{G} \rightarrow O_{d}$ is simply the inclusion mapping.

THEOREM 4. The above problem has a universal solution, denoted $A_{\otimes_{\mu}}$ $O_{d}$, where we write $A \otimes_{\mu} C$ for $\pi(A)_{\zeta}(C) . A \otimes_{\mu} O_{d}$ carries a strongly continuous action $\tilde{\alpha}$ of $G$, the dual action, with $\tilde{\alpha}_{g}\left(A \otimes_{\mu} C\right)=A \otimes_{\mu} \alpha_{g}(C)$. The fixed-point algebra for this action is $A \otimes_{\mu} I$. The closed $G$-invariant ideals in $A \otimes_{\mu} O_{d}$ are in 1-1 correspondence with the closed $\rho$-stable ideals in the fixed-point algebra.

If, in particular, we regard the mapping $\pi$ in (9) as a Hilbert space representation, the universality property in Theorem 4 shows that $A \otimes_{\mu} B$ is the appropriate generalization of the notion of covariance algebra to our context.

The $C^{*}$-system $\{B, G, \alpha\}$ of Theorem 1 is canonically isomorphic to $\left\{A \otimes_{\mu}\right.$ $\left.O_{d}, G, \tilde{\alpha}\right\}$ since, if we take $O_{d}$ to be the $C^{*}$-subalgebra generated by $H \subset B$, $\mu$ is just the inclusion $O_{G} \subset B^{\alpha}=A$. Although we are not given the group $G$ in the setting of Theorem 2, the next result allows us to apply the cross product construction with $G$ replaced by $\mathrm{SU}(d)$.

THEOREM 5. Given $A, \rho, \varepsilon_{\rho}$ and $R_{\rho}$ as in Theorem 2, there is a unique monomorphism $\mu_{0}: O_{\mathrm{SU}(d)} \rightarrow A$ with

$$
\begin{aligned}
\mu_{0}(S) & =R_{\rho}, \\
\mu_{0}(\theta(p)) & =\varepsilon_{\rho}(p), \quad p \in \mathbf{P}_{\infty}, \\
\rho \circ \mu_{0} & =\mu_{0} \circ \sigma .
\end{aligned}
$$

Writing $\tilde{B}$ for $\mathcal{A} \otimes_{\mu_{0}} O_{d}$ and $\tilde{\pi}(A)$ for $A \otimes_{\mu_{0}} I$, then $\tilde{\pi}(\mathcal{A})=\tilde{B}^{\tilde{\alpha}}$ and $\tilde{\pi}(\mathcal{A})^{\prime} \cap \tilde{B}=$ $\tilde{B}^{\prime} \cap \tilde{B}$.

Thus the $C^{*}$-system $\{\tilde{B}, \mathrm{SU}(d), \tilde{\alpha}\}$ satisfies all the requirements of Theorem 2 except that the relative commutant of the fixed-point algebra does not 
reduce to the complex numbers. A simple general procedure [7] leads to the desired result: since $\tilde{\alpha}$ acts ergodically on the centre of $\tilde{B}, \operatorname{SU}(d)$ acts transitively on its spectrum. Every point $\phi$ in that spectrum determines a stabilizer $G_{\phi} \subset \mathrm{SU}(d)$, and if $J_{\phi}$ denotes the closed ideal of $\tilde{B}$ generated by $\operatorname{Ker} \phi$ and $\eta_{\phi}$ denotes the corresponding quotient homomorphism of $\tilde{B}$ onto $\tilde{B} / J_{\phi}$, then

THEOREM 6. The $C^{*}$-system $\{B, G, \alpha\}$ obtained by setting $B=\tilde{B} / J_{\phi}$, $G=G_{\phi}$ and defining $\alpha$ by $\alpha_{g} \circ \eta_{\phi}=\eta_{\phi} \circ \tilde{\alpha}_{g}, g \in G_{\phi}$, has the properties described in Theorem 2, where $\pi=\eta_{\phi} \circ \tilde{\pi}$.

By the comments following Theorem 4, Theorem 6 provides us with an extension of $\mu_{0}$ to a monomorphism $\mu: O_{G} \rightarrow A$ for which $\mu\left(\sigma^{r}, \sigma^{s}\right)=$ $\left(\rho^{r}, \rho^{s}\right), r, s \in \mathbf{N}_{0}$. This makes the pair $(\mu, G)$ unique up to conjugation in $\mathrm{SU}(d)$; this surjectivity of $\mu$ on intertwiners reflects condition (d) and singles out our special class of cross products.

Our main theorem, Theorem 2, can be generalized to treat a semigroup $\Delta$ of endomorphisms; under an appropriate analogue of our assumptions we can conclude that $A$ is the fixed-point algebra of a dual $C^{*}$-system $\{B, G, \alpha\}$, where $G$ is a compact group which is now not necessarily a Lie group.

This has applications to Quantum Field Theory when $A$ is the $C^{*}$-algebra of local observables whose local structure determines a semigroup $\Delta_{r}$ of endomorphisms associated with superselection sectors [3]. For each $\rho \in \Delta_{r}$, there is a canonical unitary representation $\varepsilon_{\rho}$ of $\mathbf{P}_{\infty}$ in $A$ describing the (particle) statistics. As this structure is deduced from basic principles, it provides a general analysis of the possible statistics in particle physics: the support of $\varepsilon_{\rho} \mid \mathbf{P}_{n}$ corresponds to the set of Young tableaux with $n$ squares and at most $d$ rows, when $\rho$ is said to have para-Bose statistics of order $d$, or with at most $d$ columns, para-Fermi statistics of order $d[\mathbf{3}, \mathbf{8}]$.

The results announced here allow us to conclude that there is a compact group $G$, the gauge group of the first kind, whose representation theory describes the superselection structure, and an action of $G$ on a $C^{*}$-algebra $₹$ of fields with normal commutation relations.

Another application is to the duality theory of compact groups. These results allow one to recognize the representation theory of a compact group when it is given concretely in terms of endomorphisms of a $C^{*}$-algebra and their intertwining operators, but it is possible to go further and strengthen the Tannaka-Krein duality theorem, by characterizing the abstract category of representations of a compact group.

We also envisage developments of the present analysis with applications to $K$-theory, duality for cross products, and the Connes spectrum.

\section{REFERENCES}

1. S. Doplicher, R. Haag and J. E. Roberts, Fields, observables and gauge transformations. I, Comm. Math. Phys. 13 (1969), 1-23.

2. S. Doplicher and J. E. Roberts, Fields, statistics and non-abelian gauge groups, Comm. Math. Phys. 28 (1972), 331-348. 
3. S. Doplicher, R. Haag and J. E. Roberts, Local observables and particle statistics. I, II, Comm. Math. Phys. 23 (1971), 199-230; ibid. 35 (1974), 49-85.

4. J. Cuntz, Simple $C^{*}$-algebras generated by isometries, Comm. Math. Phys. 57 (1977), 173-185.

5. _ K-theory for certain $C^{*}$-algebras. II, J. Operator Theory 5 (1981), 101-108.

6. J. E. Roberts, Cross products of von Neumann algebras by group duals, Sympos. Math. 20 (1976), 335-363.

7. S. Doplicher and J. E. Roberts, A remark on compact automorphism groups of $C^{*}$-algebras (preprint).

8. D. Buchholz and K. Fredenhagen, Locality and the structure of particle states, Comm. Math. Phys. 84 (1982), 1-54.

Dipartimento di MATEMATiCA, UniVERsità di Roma "LA SAPIEnZA", 1-00185 ROMA, ITALY

UNiversität OSNABRÜCK, FACHBEREICH PhysiK, D-4500 OSNABRÜCK, FeDERAL REPUBLIC OF GERMANY 